\title{
PULSATILE FLOW IN THE PULMONARY CIRCULATION: A CINEFLUOROSCOPIC STUDY
}

\author{
BY \\ A. R. CHRISPIN AND R. E. STEINER \\ Department of Diagnostic Radiology, Hammersmith Hospital, Postgraduate Medical School of London
}

Received January 8, 1964

This paper describes a radiological technique which can be used to demonstrate and analyse pulsatile blood flow in the pulmonary vascular bed.

\section{Methods ANd Patients Studied}

A mechanical pressure injector of the type described by Jacobson (1963) was used. Contrast medium was injected from a metal syringe through an end-hole No. 7 or 8 American gauge cardiac catheter that had been passed into a pulmonary artery. Before an injection the catheter was filled by hand from a separate syringe interposed between the metal syringe and the catheter. Each injection was timed with a very accurate stopwatch. By varying the force exerted by the ram against the piston of the metal syringe the duration of the injection could be altered. The rate of injection of contrast from the end-hole of the catheter was proved by experiments using repeated test injections with concommitant timing to be constant except for the very short periods at the very beginning and end of the injection. It was also shown experimentally that at pressures normally encountered in the pulmonary circulation the rate of injection of contrast medium was not affected by the pressure in the vicinity at the end-hole of the catheter. This was done by test injections into the base of a column of water, the height of which was varied to produce hydrostatic pressures comparable to those in the pulmonary circulation.

For this investigation it was arranged that $4 \cdot 5-5 \mathrm{ml}$. of 65 per cent Hypaque were injected within a period of 1.5 to 3 seconds. This permitted observations of the pulmonary circulation during a number of heart beats which were monitored simultaneously on the electrocardiogram. Each pulmonary artery injection was filmed by using a $9 \mathrm{in}$. Phillips image amplifier and $35 \mathrm{~mm}$. Arriflex cine-camera at a film rate of approximately 42 exposures per second.

The pulmonary blood flow in 11 patients was investigated by cine-angiography (Table I). Contrast medium was injected into the main pulmonary artery, lobar pulmonary arteries, segmental arteries, and in the "pre-wedge" position in the vascular lobule (Jacobson, 1963). A total of 15 injections was made, 2 into the main branch of the right pulmonary artery, 3 into a lobar artery, 4 into a small segmental lobar artery, 5 in the "off wedge position" in a similar position, and one in the wedge position. All patients were lying at rest and breathing quietly throughout the examination which was made during cardiac catheterization and before angiocardiography. Only rarely did a patient give a slight cough towards the end of the injection but no other sequelae were observed. Heart rate and rhythm were undisturbed by this procedure.

\section{INTERPRETATION OF FILM RECORD}

The cine films were analysed on a Tage Arno RG 35 projector which permits the examination of individual frames as well as projection at varying speeds. The film records were accurately correlated with the patient's electrocardiogram taken during the examination. The following obser- 
TABLE I

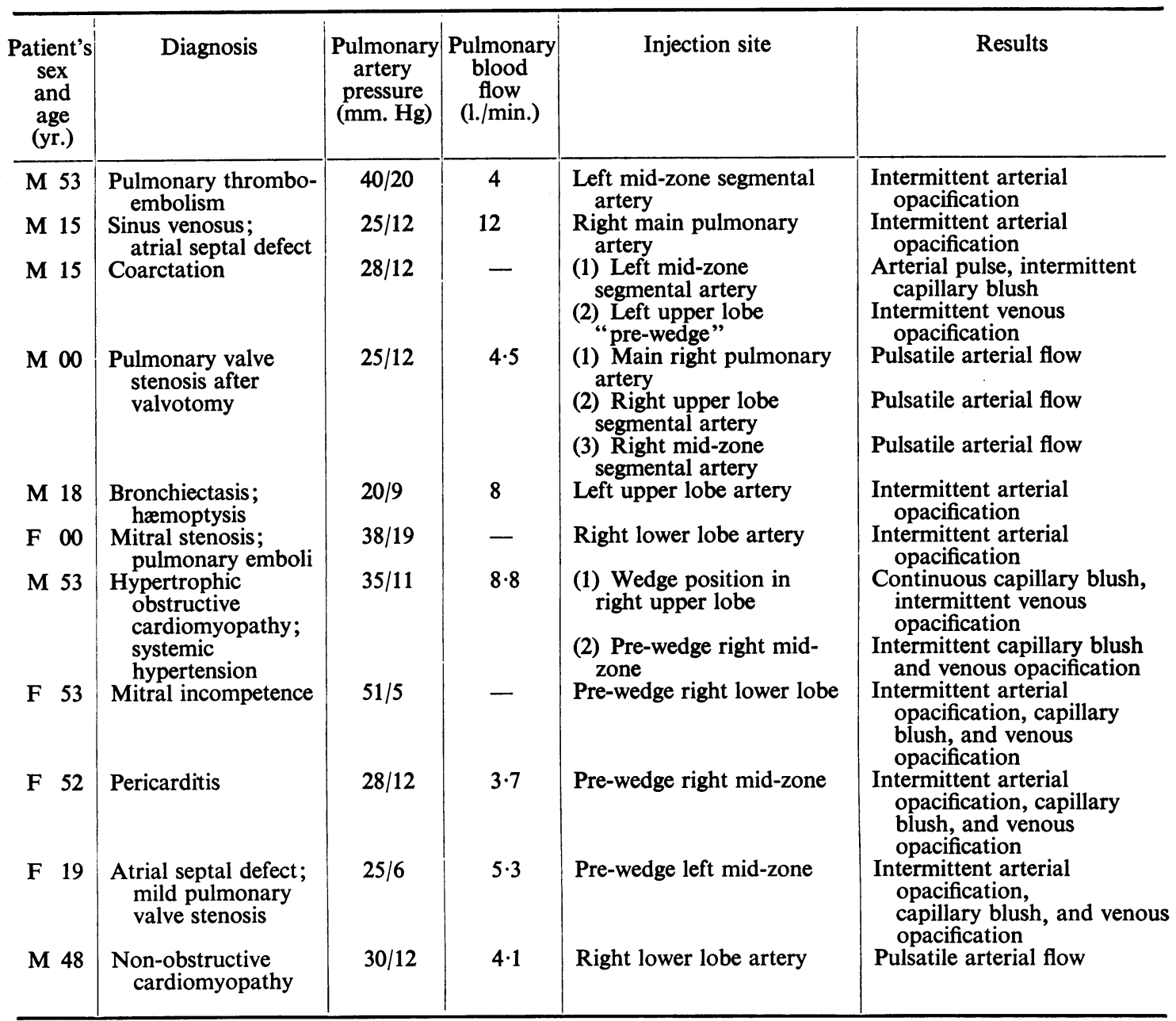

vations were made: in 14 injections an intermittent opacification of the pulmonary arterial bed was seen. In 3 patients who had a "pre-wedge" injection, a fluctuating density of the smallest pulmonary vessels including arterioles, capillary bed, and venules could be observed, and in 6 patients intermittent opacification of some small pulmonary veins was noticed.

To illustrate some of our findings angiograms of 3 patients with normal pulmonary artery pressure and blood flow are described in addition to the angiograms of one patient who had an atrial septal defect with slight pulmonary valve stenosis resulting in a raised pulmonary blood flow and a normal pressure (Fig. 1, 2, 3, and 4).

Lobar and Segmental Artery Injection. Two separate phases can be seen.

(1) Vascular opacification: After the start of the injection the lumen of the artery at the catheter tip opacifies (Fig. 1a, 2a). Arteries distal to the catheter tip subsequently opacify but there is also a little spread of contrast proximal to the point of injection (Fig. 1b, c, e). The density of the contrast in the arteries is high considering the slow rate of injection, and it is clear therefore that the contrast medium is not being greatly diluted.

These findings suggest that there is virtually no blood flow in these vessels during this phase of the examination. 

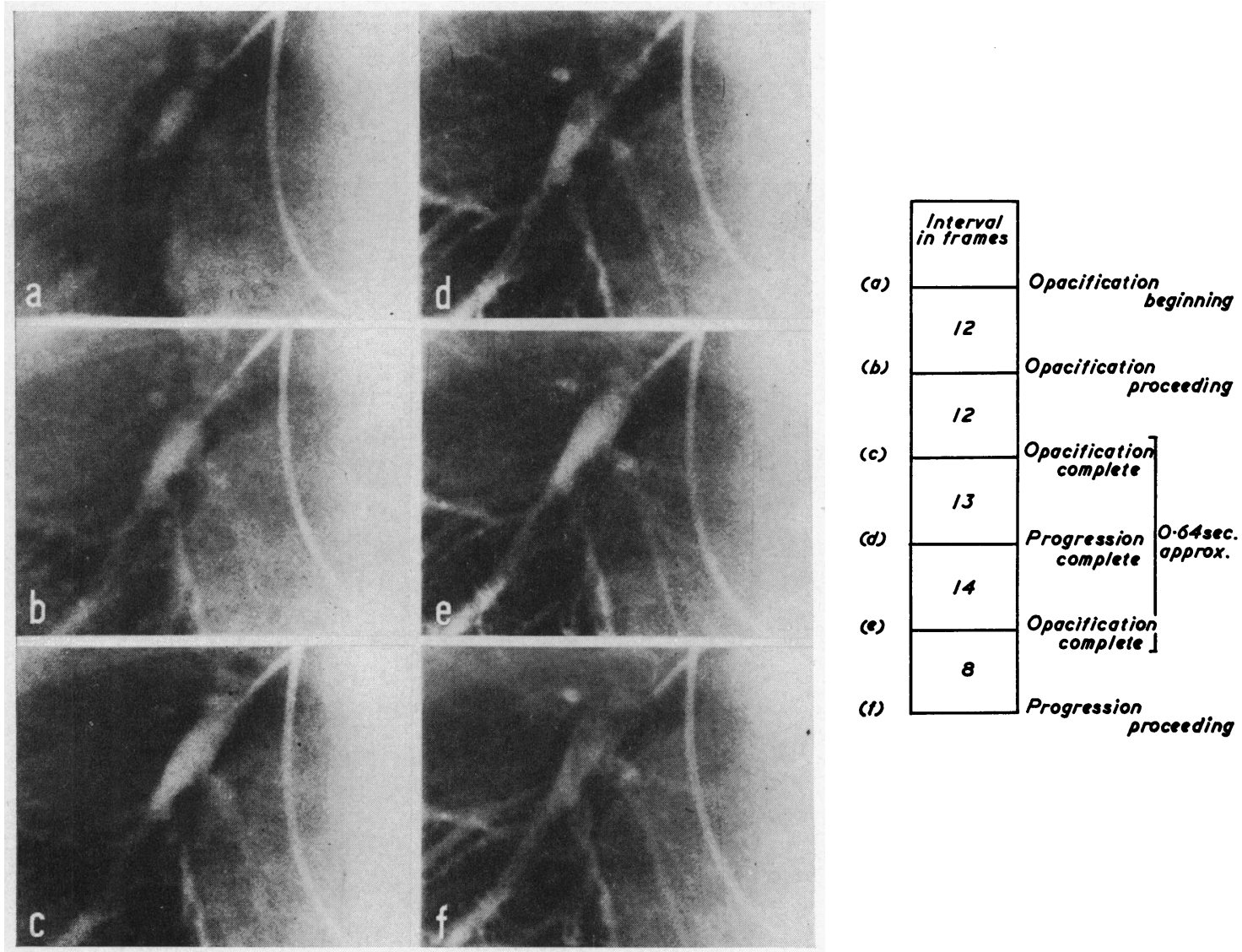

Fig. 1.-Man, 48 years, with cardiomyopathy. Pulmonary arterial pressure 25-30/12-8 $\mathrm{mm}$. $\mathrm{Hg}$, mean $14 \mathrm{~mm}$. $\mathrm{Hg}$, flow $4 \cdot 1 \mathrm{l} . / \mathrm{min}$. Right lower lobe artery injection through No. 8 cardiac catheter. $5 \mathrm{ml} .65$ per cent Hypaque injected over $1.48 \mathrm{sec}$. Cardiac cycle $0.6 \mathrm{sec}$. on concurrent electrocardiogram. Note in (f) the lamination of the contrast along the wall of the artery due to high axial flow rate.

(2) Progression of contrast medium: Quite suddenly the contrast medium in arteries in the vicinity of the end-hole of the catheter begins to move rapidly into smaller pulmonary arterial branches (Fig. 1d, f; Fig. 2b). In spite of the continuous injection of contrast medium the arteries which were initially opaque empty rapidly, and the previous extensive opacification of these vessels is no longer seen. The contrast which is being injected is rapidly diluted by blood. These findings indicate a high rate of blood flow in these arteries at this time. Early in this phase it can be seen that the contrast in the centre of the lumen of the artery moves more rapidly than at the margin of the lumen adjacent to the vessel wall (Fig. 1d, f). This phenomenon of a high axial flow rate is a well-recognized feature of systemic arterial blood flow.

The cycle of opacification and progression of contrast medium occurs at the same rate as the cycle of ventricular diastole and systole (determined from the concurrent electrocardiogram). In a number of patients when left lung studies were carried out it was sometimes possible to see on the cine film both ventricular contraction and the forward movement of contrast medium in the vascular bed. It could then be seen that ventricular systole was very rapidly followed by movement of the contrast through the pulmonary arteries. 


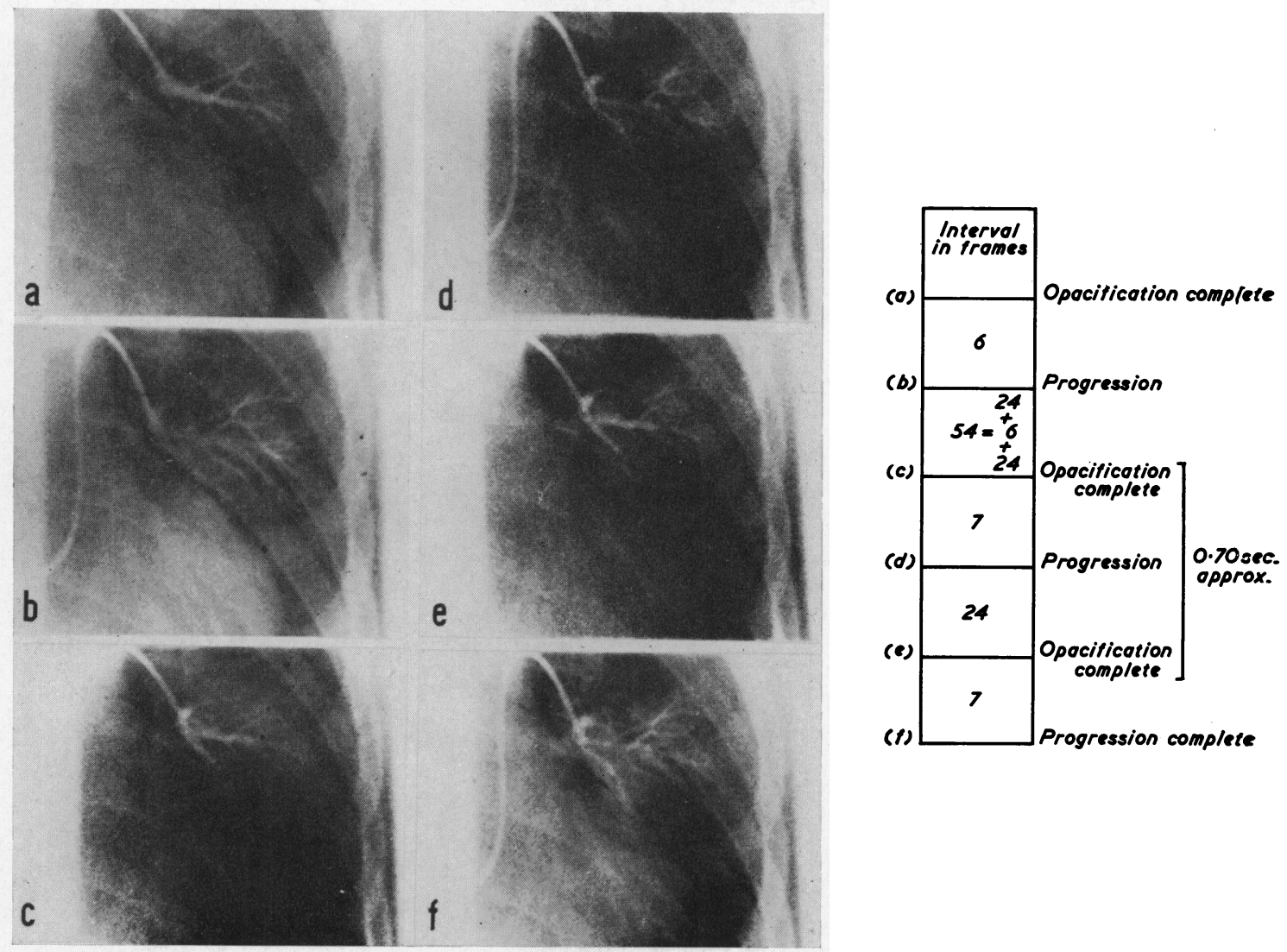

FIG. 2.-A youth of 15 years with coarctation of aorta. Pulmonary arterial pressure $28 / 12 \mathrm{~mm}$. $\mathrm{Hg}$, mean $18 \mathrm{~mm}$. Hg. Left mid-zone segmental artery injection through No. 7 cardiac catheter. $4.5 \mathrm{ml}$. 65 per cent Hypaque injected over $3.05 \mathrm{sec}$. Cardiac cycle $0.68 \mathrm{sec}$. on concurrent electrocardiogram. Note increased density of blush due to contrast in small vessels after each heart beat.

These observations show the highly pulsatile nature of pulmonary blood flow in the large and smaller segmental pulmonary arteries.

The Pre-wedge Injection. If a No. 7 or 8 cardiac catheter is wedged in a pulmonary artery it occludes a vessel of $2 \cdot 5-3 \mathrm{~mm}$. calibre. This type of vessel usually tapers gradually, giving off many lateral arborizing branches which supply the capillary bed of a lobule (Jacobson, 1963). To obtain a satisfactory pre-wedge angiogram the catheter was first wedged and then withdrawn to a position just proximal to the wedge position. In the pre-wedge position the pulmonary artery pressure can be recorded. From injections in this position it was possible to study flow in small arteries, the capillary bed, and small pulmonary veins (Fig. 3 and 4).

The calibre of the smallest arteries that can be indentified individually in the film records probably lies in the 100-500 $\mu$ range. On analysis of the films a cycle of arterial opacification and progression of contrast, related to ventricular diastole and systole, can be seen. The central artery of the vascular lobule and its lateral branches exhibit pulsatile flow similar to that in lobar and segmental arteries (Fig. 3a, b, c). Flow of contrast medium through the smallest vessels, including the capillaries, produces a blush of contrast medium in which individual vessels cannot be discerned. During 

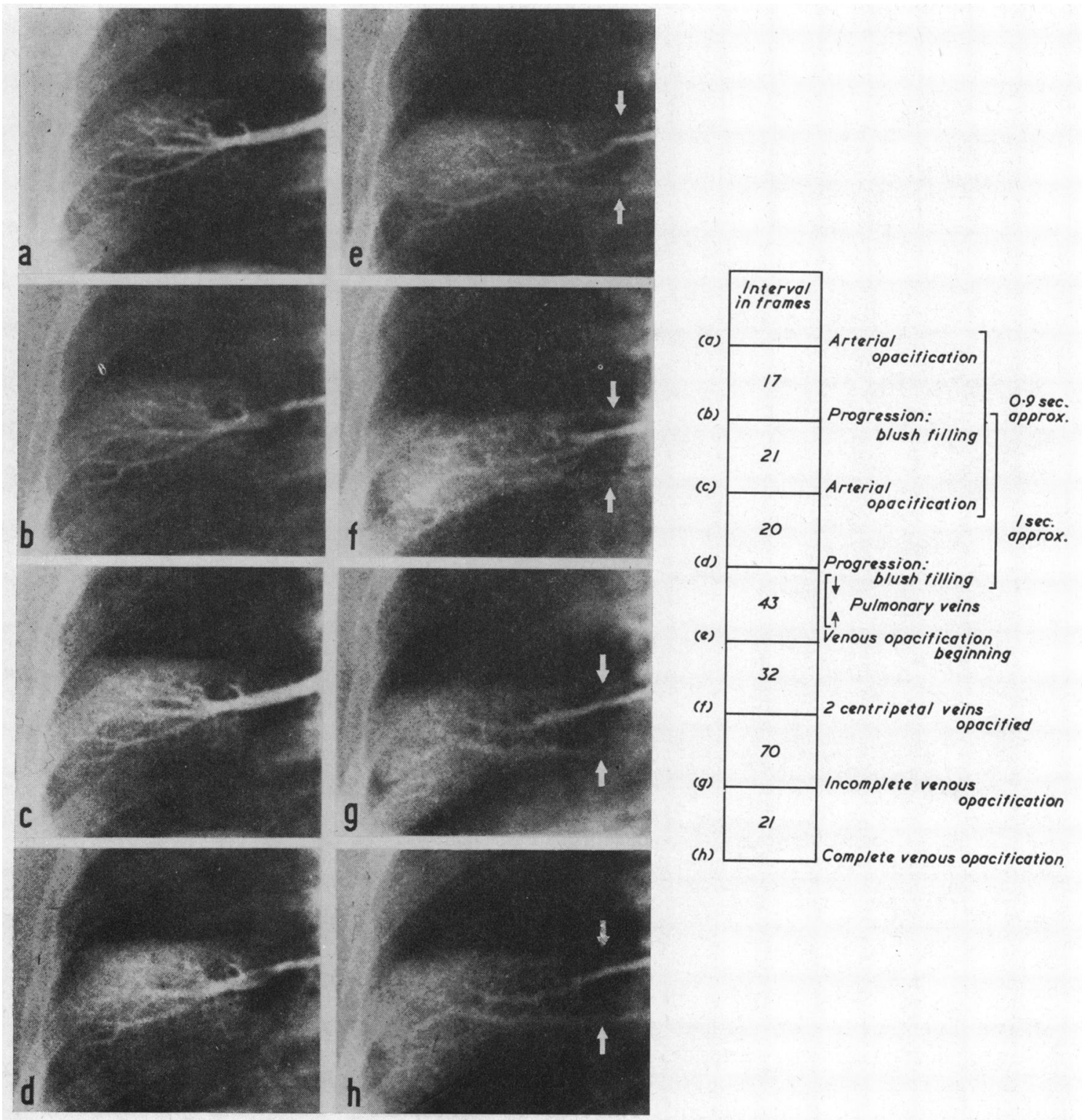

Fig. 3.-A woman of 52 years with pericarditis. Pulmonary artery pressure $28 / 12 \mathrm{~mm}$. $\mathrm{Hg}$, mean $17 \mathrm{~mm}$. Hg; flow 3.7 l./min. Right mid-zone "pre-wedge" through No. 8 cardiac cathether. $4.5 \mathrm{ml}$. 65 per cent Hypaque injected over $2.4 \mathrm{sec}$. Cardiac cycle $1.0 \mathrm{sec}$. on concurrent electrocardiogram.

the injection period the density of the blush increases intermittently and at the same rate as the heart rate (Fig. 3c, d, e, f). This suggests that the blood flow through these small vessels is pulsatile.

In the venous phase of the pre-wedge angiogram, two main lobular veins are commonly seen carrying blood and contrast medium away from the periphery of the lung lobule. Each lobular vein joins with similar veins from neighbouring lobules to form a centripetal vein carrying blood to 

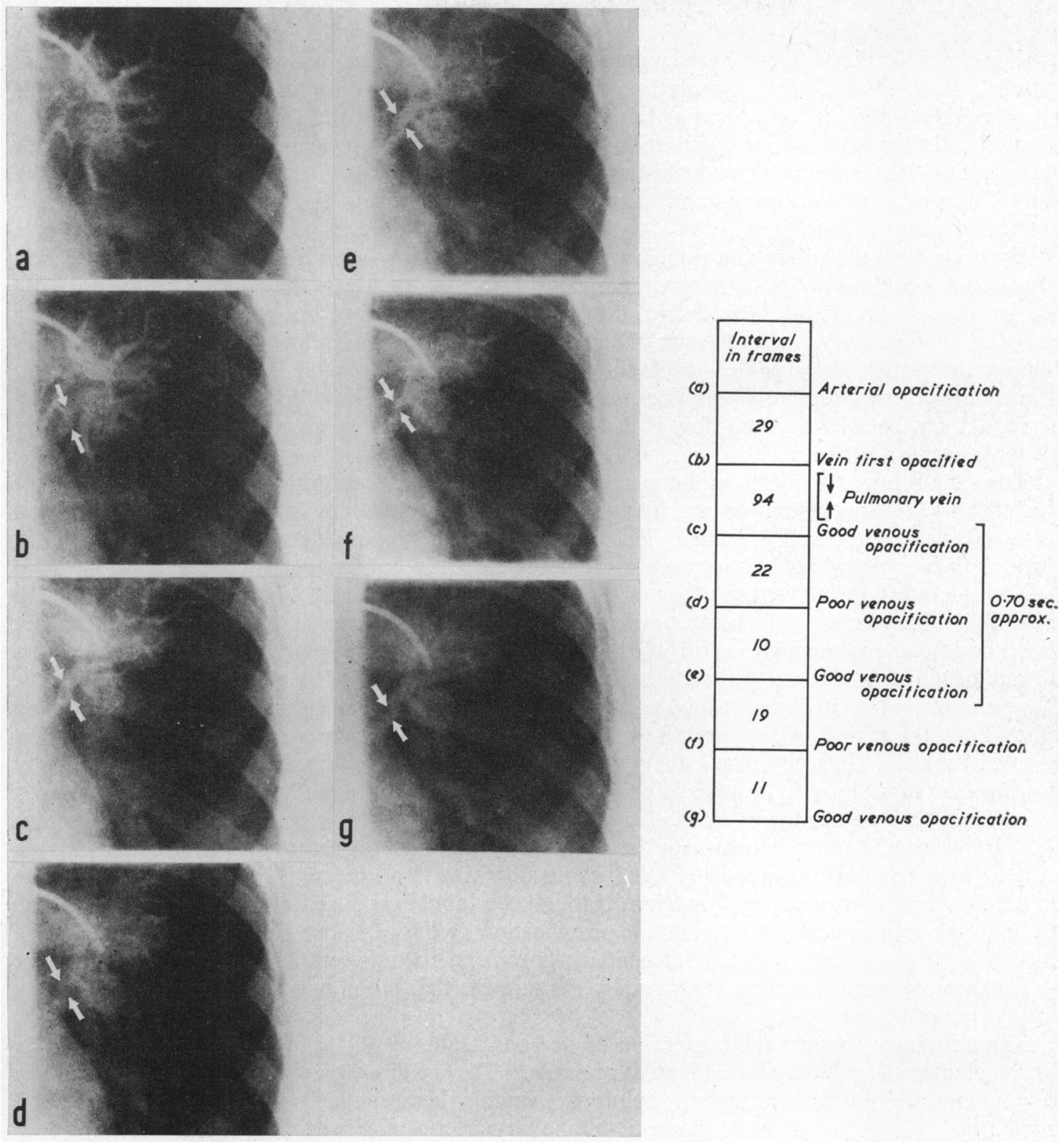

FIG. 4.-Cine-angiograms from 19-year-old woman with atrial septal defect with mild pulmonary valve stenosis. Left-to-right shunt at atrial level. Gradient at pulmonary valve $25 \mathrm{~mm}$. $\mathbf{H g}$; pulmonary arterial pressure $25 / 6 \mathrm{~mm}$. $\mathrm{Hg}$; flow $5 \cdot 21$. $/ \mathrm{min}$. Pulmonary systemic flow $=1 \cdot 9: 1$. Left mid-zone "pre-wedge" injection through No. 7 cardiac catheter. $4.5 \mathrm{ml}$. 65 per cent Hypaque injected over $2.48 \mathrm{sec}$. Cardiac cycle $0.7-0.8 \mathrm{sec}$. on concurrent electrocardiogram.

the lobar vein and the lung root. Following a pre-wedge injection the lobular veins opacify and maintain a steady level of density until the blush fades as the contrast leaves the lobule. This indicates that blood and contrast are well mixed by the time this point in the circulation is reached. The centripetal veins opacify intermittently at the same rate as the heart rate, and this seems to be best explained as a result of pulsatile venous flow (Fig. 3e, f, g, h; Fig. 4b, c, d, e). 


\section{Discussion}

It is clear that blood flow past the pulmonary valve is intermittent and related to right ventricular systole. With the aid of the whole body plethysmograph and using a modification of Krogh's nitrous oxide technique for measuring lung blood flow in man, Lee and DuBois (1955) deduced that blood flow in the capillaries was pulsatile. Similar findings by Harris and Mills, quoted by Harris and Heath (1962), support this conclusion. However, Rigatto and Fishman (1960) have doubted the validity of such conclusions and they have drawn attention to the purely mechanical factors associated with the heart beat.

By using a cinefluorographic technique and a relatively slow but uniform continuous injection of contrast medium into pulmonary arteries it is possible to investigate the flow at various points in the pulmonary circulation. In arteries down to $100-500 \mu$ calibre this can be seen to be highly pulsatile. When arteries near the site of injection are opacified blood flow is minimal: this phase is related to ventricular diastole. Following systole rapid progression of contrast into smaller arteries indicates a transiently high flow. At such times of high blood flow in lobar and segmental arteries it can be seen that the flow is fastest in the middle of the lumen and therefore not only pulsatile but also laminar.

During a pre-wedge injection of contrast medium there is an intermittently increasing density of the blush which results from contrast filling the small vessels of the lung lobule. This can be related to ventricular systole and diastole. This finding strongly suggests that there is a pulsatile flow through the very small pulmonary vessels, including the arterioles, capillaries, and venules.

Consideration of the venous phase of the angiogram seems to indicate a pulsatile blood flow in small pulmonary veins. The presence of a cardiac catheter in the central artery (partly obstructing free blood flow) and the high viscosity of the contrast medium are probably responsible for the overall rate of blood flow through the injected lobule being slower than that in adjacent lobules. It is, therefore, likely that in adjacent lobules, where there is no contrast medium or catheter obstructing blood flow, the period of increased flow begins a little earlier and lasts a little longer than in the injected lobule. Thus contrast leaving the lobular veins of the injected lobule joins a rapidly flowing stream of blood from other lobular veins. When flow from the injected lobule falls there is still sufficient flow from the other lobular veins to carry contrast through the centripetal veins into the lobar veins and the lung root. In the one patient where contrast medium was injected with the catheter in the wedge-position a pulsatile venous flow was also observed. It seems likely that the continuous injection of contrast medium through the lobule produced a steady flow in the veins draining the lobule, and yet intermittent opacification of the centripetal veins was observed; this must have been produced by the intermittent flow from the adjacent lobules. The intermittent appearance of contrast in these centripetal veins suggests that blood flow in the smaller pulmonary veins is pulsatile.

These findings support the contention of Lee and Dubois (1955) that the pulmonary vascular flow, including the capillary blood flow, is pulsatile. The cinefluorographic technique described has shown that the blood flow through the pulmonary vascular bed is pulsatile in arteries, capillaries, and small veins. Even though the large elastic pulmonary arteries normally change in calibre by about 10 per cent during the cardiac cycle (Brandt and Chrispin, 1963), the recoil in these arteries is apparently insufficient to produce a steady flow in the small pulmonary vessels. The present investigation has been limited to patients with normal or only slightly raised pulmonary artery pressures, but it is proposed to study the pulmonary vascular bed in patients with a high pulmonary artery pressure and raised pulmonary vascular resistance using the same technique.

\section{SUMMARY}

A cinefluorographic technique with a slow continuous injection of contrast medium has been used to investigate the nature of the pulmonary blood flow. The findings are compatible with a pulsatile flow in pulmonary arteries, capillaries, and small pulmonary veins. 
We wish to thank Professor J. F. Goodwin for his help and encouragement with this investigation. We must also acknowledge our debt to Professor G. Jacobson who developed the injection pump.

\section{REFERENCES}

Brandt, P., and Chrispin, A. (1963). Pulmonary artery pulsation in ventricular septal defect. Clin. Radiol., 14, 333.

Harris, P., and Mills, R. (unpublished) quoted by P. Harris, and D. Heath (1962). The Human Pulmonary Circulation, p. 62 . Livingstone, Edinburgh.

Jacobson, G. (1963). Peripheral pulmonary (wedge) arteriography. A standardised technique for the single film arteriogram. Clin. Radiol., 14, 326.

Lee, G. de J., and DuBois, A. B. (1955). Pulmonary capillary blood flow in man. J. clin. Invest., 34, 1380.

Rigatto, M., and Fishman, A. P. (1960). The pulsatile nature of the pulmonary capillary blood flow. J. clin. Invest., 39, 1626. 